\title{
Expeditious Multicomponent Synthesis of Xanthone Dimers ${ }^{+}$
}

\author{
Ana Bornadiego, Jesús Díaz * and Carlos F. Marcos *
}

Departamento de Química Orgánica e Inorgánica, Facultad de Veterinaria, Universidad de Extremadura. Avda. Universidad, s/n, 10003 Cáceres, Spain; bornadiegos@unex.es

* Correspondence: jdal@unex.es (J.D.); cfernan@unex.es (C.F.M.); Tel.: +34-9-2725-7158 (C.F.M.); Fax: +34-9-2725-7110 (C.F.M.)

+ Presented at the 22nd International Electronic Conference on Synthetic Organic Chemistry, 15 November15 December 2018; Available Online: https://sciforum.net/conference/ecsoc-22.

Published: 14 November 2018

\begin{abstract}
Xanthones are a type of compound widely found in many natural products from plants, fungi, and lichens and are considered privileged structures. Frequently, xanthones occur in nature as dimers, which often exhibit singular and potent biological effects. Although diverse methods for the synthesis of monomeric xanthones are known, dimeric xanthones remain synthetically challenging targets. Reported syntheses of dimeric xanthones are very scarce, and invariably involve a large number of synthetic steps. We have recently developed a multicomponent synthesis of xanthones starting from 3-carbonylchromones, isocyanides, and dienophiles. Here we report a similar one-pot tandem procedure, involving a [4+1]-[4+2] cycloaddition, that readily affords dimeric xanthones and dihydroxanthones, which are structurally similar to bioactive ergochromes.
\end{abstract}

Keywords: multicomponent reactions; isocyanides; natural products; heterocycles

\section{Introduction}

Xanthones are "privileged structures" that have shown a wide variety of biological activities [1]. Particularly, xanthone dimers are abundant metabolites found in many plants, fungi, and lichens, and are valued compounds due to their unique biological activities [2]. For example, in contrast with the activity of xanthone monomers, which usually target topoisomerase II [3], xanthone dimers, such as secalonic acids (Figure 1) [4], have been investigated as anticancer agents due to their selective inhibition of DNA topoisomerase I (Topo I) $[5,6]$.

It is known that small chemical changes can modulate, or drastically modify, the biological activities of dimeric xanthones [6]. For example, partial hydrogenation of one of the aromatic rings [7], and the presence of hydroxy [8] or amino [3] polar groups in the xanthone core can determine their biological profile. It is thus crucial to find new synthetic methods that allow for obtaining diverse dimeric xanthones rapidly and efficiently.

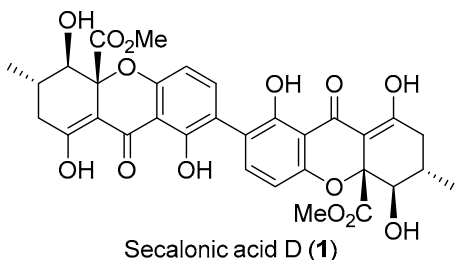

Secalonic acid D (1)

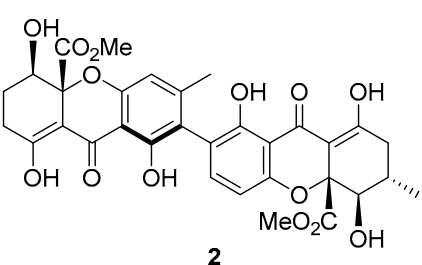

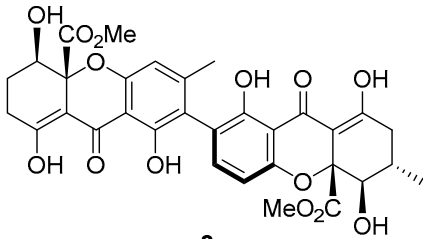

Figure 1. Natural xanthone dimers with activity as Topo I poisons $[5,6]$. 
Multicomponent reactions (MCR) are atom economic and highly convergent processes, in which three or more starting materials react to give complex products $[9,10]$. We have recently reported the multicomponent synthesis of anilines from $\alpha, \beta$-unsaturated keto-esters, isocyanides and phthalimides [11]. This novel methodology was successfully applied to the synthesis of a variety of 4-aminoxanthones [12] and dihydroxanthones (Scheme 1) [13].

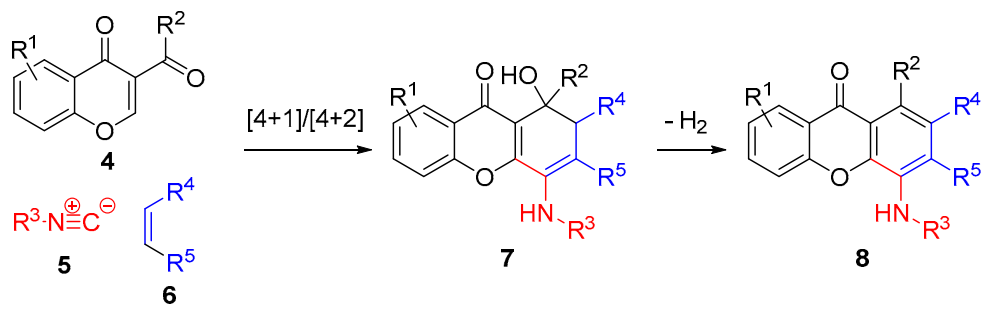

Scheme 1. Multicomponent synthesis of dihydroxanthones and xanthones.

The flexibility and experimental simplicity of this multicomponent reaction make it ideal for the synthesis of dimeric derivatives. Thus, here we report a novel strategy for the synthesis of dimeric xanthones and dihydroxanthones by a double multicomponent reaction of 3-carbonylchromones, isocyanides, and dienophiles.

\section{Results and Discussion}

Our strategy for the synthesis of dimeric xanthones is based in the simultaneous building of both xanthone units from the corresponding 3-carbonylchromone units. Thus, according to the proposed retrosynthetic plan (Scheme 2), a dimeric carbonylchromone (10) should be used as starting material.

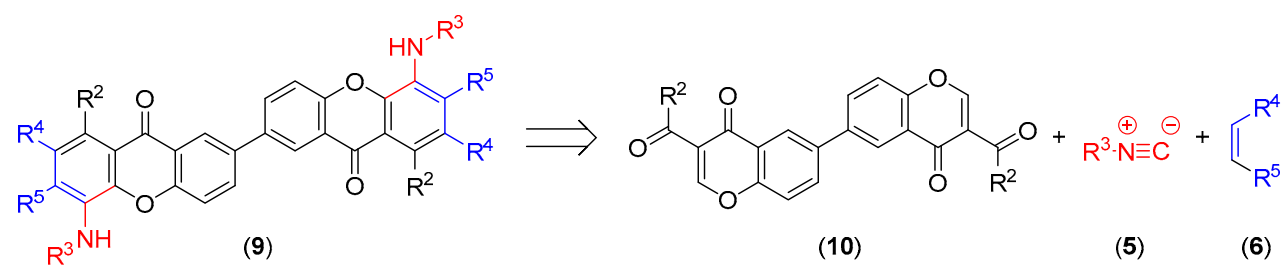

Scheme 2. Retrosynthesis of xanthone dimers.

Bischromone (10) could be synthesized from readily available bisphenol (11). Thus, hydroxyl groups were acetylated by treatment with acetic anhydride and pyridine. Then, acid catalyzed Fries rearrangement [14] led almost quantitatively to 1,1'-(4,4'-dihydroxy-[1,1'-biphenyl]-3,3'-diyl)bis(ethan-1-one) (12) [15]. This was subjected to aldol condensation with dimethylformamide dimethyl acetal (13) under microwave irradiation [16] to give enaminone 14. Finally, reaction with methyl 2-chloro-2-oxoacetate (15) and pyridine, according to the modified procedure of Iaroshenko and Langer [17], produced the desired dimeric chromone (10a) in good yield, as shown in Scheme 3. 

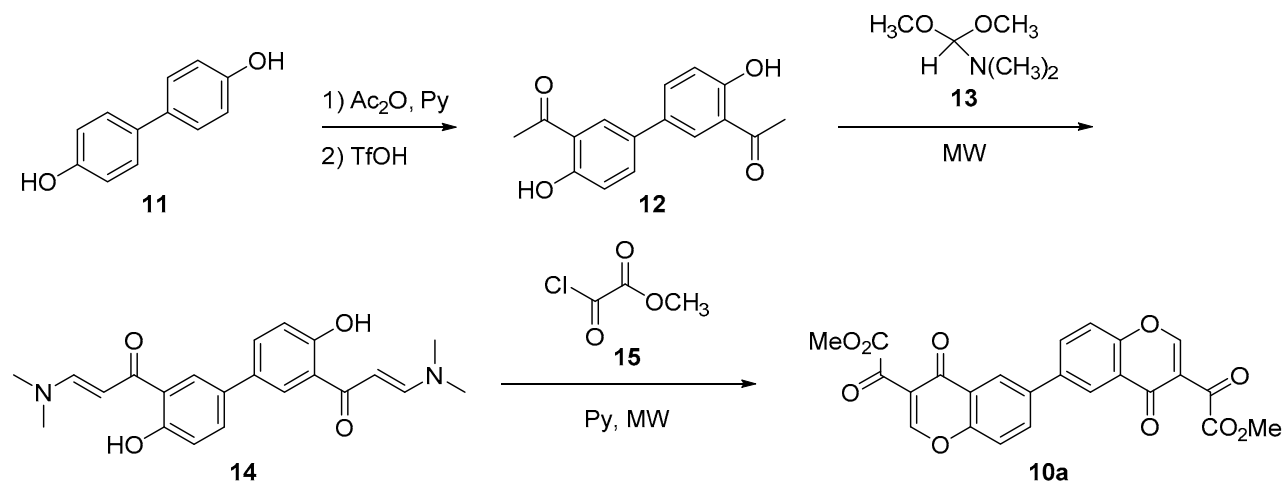

Scheme 3. Synthesis of dimeric chromone (10a).

Bischromone (10a) was used to prepare dimeric xanthone and dihydroxanthone derivatives. Thus, the reaction with cyclohexyl isocyanide (5a) and $N$-phenylmaleimide (6a), in refluxing THF, after $6 \mathrm{~h}$, successfully afforded xanthone dimer (16a) in a 79\% yield (49\% overall yield from bisphenol 11; Scheme 4).
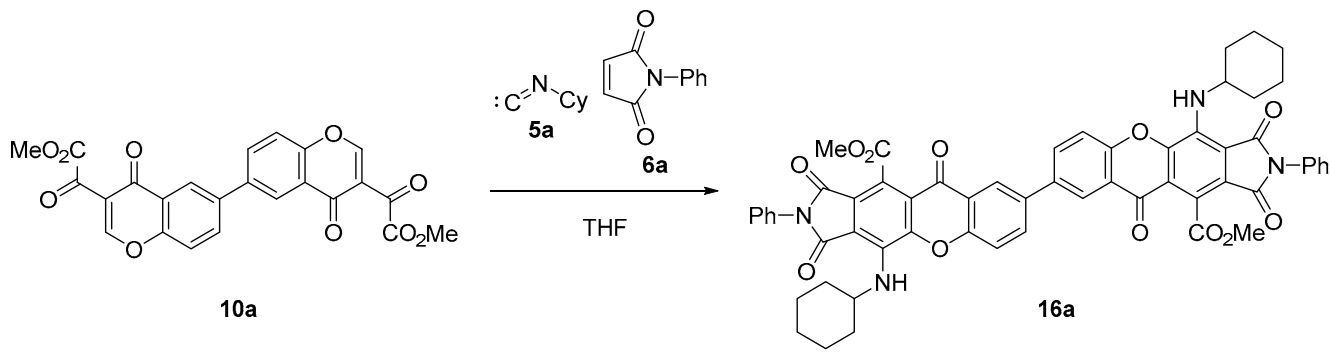

Scheme 4. Synthesis of dimeric xanthone (16a).

On the other hand, the reaction of bischromone (10a) with cyclohexyl isocyanide (5a) and acrylonitrile (6b), under reflux in THF for $6 \mathrm{~h}$, successfully produced dihydroxanthone dimer (17b) in an $89 \%$ yield, as shown in Scheme 5.
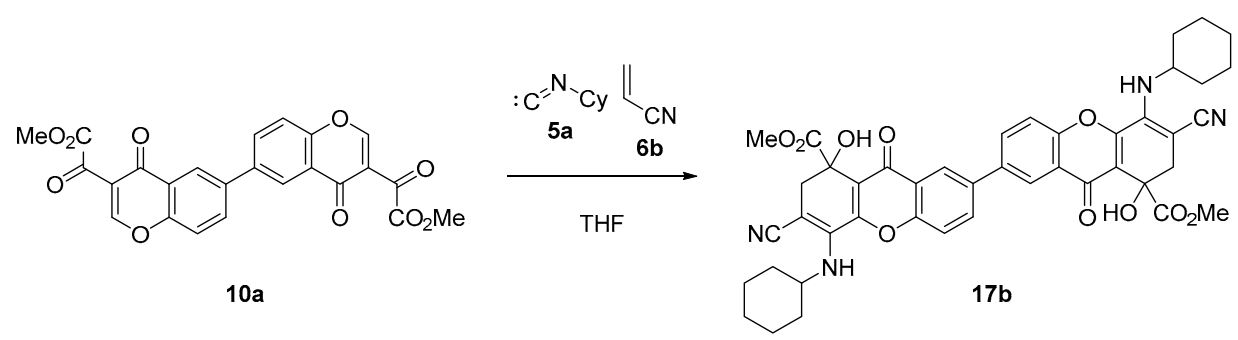

Scheme 5. Synthesis of dimeric dihydroxanthone (17b).

The reaction must take place through a double tandem [4+1]/[4+2] cycloaddition, according to the mechanism proposed in Scheme 6. The first step is a [4+1] cycloaddition of isocyanide (5) with the $\alpha, \beta$-unsaturated carbonyl on both chromone rings in dimeric chromone (10), to give an intermediate bisiminolactone (18). This would tautomerize to bisaminofuran (19) that, in turn, would undergo [4+2] cycloaddition reaction with the dienophile to give Diels-Alder adduct (20). The assistance of the nitrogen lone pair on the resulting 7-oxabicyclo[2.2.1] heptanes would enable the in situ opening of the oxygen bridges to give the corresponding dihydroxanthones (17). With asymmetric dienophiles, such as acrylonitrile, the corresponding dihydroxanthone $\mathbf{( 1 7 b})$ is a stable product that can be easily isolated. On the other hand, when the dienophile is $N$-phenylmaleimide, 
the acidic character of hydrogen on position 2 of the dihydroxanthone facilitates the elimination of a molecule of water in the reaction conditions, directly affording fully aromatized dimeric xanthone (16a).<smiles>CCOC(=O)c1coc2ccc(-c3ccc4occ(C(=O)OC)c(=O)c4c3)cc2c1=O</smiles><smiles></smiles>

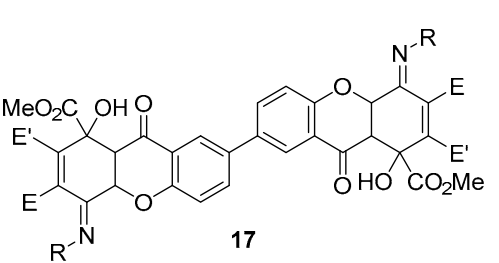
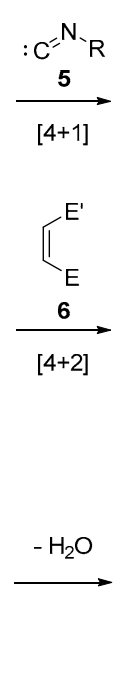<smiles>[R]N=C1OC(C(C)=O)=C2C(=O)c3cc(-c4ccc5c(c4)C(=O)C4=C(C(=O)OC)OC(=N[R])C4O5)ccc3OC12</smiles>

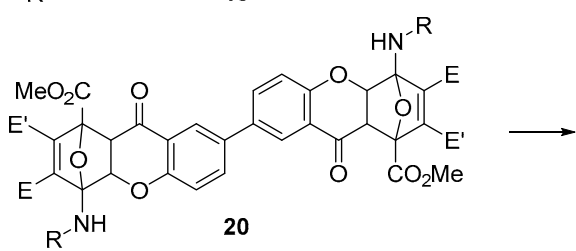<smiles>[R]Nc1c(F)c(F)c(C(C)=O)c2c(=O)c3cc(-c4ccc5oc6c(N[R])c(F)c(F)c(C(C)=O)c6c(=O)c5c4)ccc3oc12</smiles>

Scheme 6. Proposed mechanism for the synthesis of dimeric xanthones $(\mathbf{1 6}, \mathbf{1 7})$.

\section{Conclusions}

In summary, we have developed a novel, straightforward, tandem synthesis of dimeric polysubstituted 4-aminoxanthones starting from structurally simple and readily available bisphenol. The key step is a double multicomponent reaction of bischromone (10a) with an isocyanide and dienophiles. The products are available in a matter of hours and are easily isolated and purified by column chromatography. This illustrates the potential of multicomponent reactions to rapidly and efficiently build molecules of high structural complexity from very simple starting materials and constitutes the first example of a multicomponent synthesis of dimeric xanthones and dihydroxanthones.

Acknowledgments: We thank the financial support from the Junta de Extremadura and FEDER (IB16095).

\section{References}

1. Masters, K.-S.; Bräse, S. Xanthones from fungi, lichens, and bacteria: The natural products and their synthesis. Chem. Rev. 2012, 112, 3717-3776.

2. Wezeman, T.; Brase, S.; Masters, K.-S. Xanthone dimers: A compound family which is both common and privileged. Nat. Prod. Rep. 2015, 32, 6-28.

3. Minniti, E.; Byl, J.A.W.; Riccardi, L.; Sissi, C.; Rosini, M.; De Vivo, M.; Minarini, A.; Osheroff, N. Novel xanthone-polyamine conjugates as catalytic inhibitors of human topoisomerase II $\alpha$. Bioorg. Med. Chem. Lett. 2017, 27, 4687-4693.

4. Qin, T.; Porco, J.A. Total syntheses of secalonic acids A and D. Angew. Chem. Int. Ed. 2014, 53, 3107-3110.

5. Hong, R. Secalonic acid D as a novel DNA topoisomerase I inhibitor from marine lichen-derived fungus Gliocladium sp. T31. Pharm. Biol. 2011, 49, 796-799.

6. Wu, G.; Qi, X.; Mo, X.; Yu, G.; Wang, Q.; Zhu, T.; Gu, Q.; Liu, M.; Li, J.; et al. Structure-based discovery of cytotoxic dimeric tetrahydroxanthones as potential topoisomerase I inhibitors from a marine-derived fungus. Eur. J. Med. Chem. 2018, 148, 268-278.

7. Li, T.X.; Yang, M.H.; Wang, Y.; Wang, X.B.; Luo, J.; Luo, J.G.; Kong, L.Y. Unusual dimeric tetrahydroxanthone derivatives from Aspergillus lentulus and the determination of their axial chiralities. Sci. Rep. 2016, 6, 38958.

8. Wang, Q.; Ma, C.; Ma, Y.; Li, X.; Chen, Y.; Chen, J. Structure-activity relationships of diverse xanthones against multidrug resistant human tumor cells. Bioorg. Med. Chem. Lett. 2017, 27, 447. 
9. Domling, A.; Wang, W.; Wang, K. Chemistry and biology of multicomponent reactions. Chem. Rev. 2012, 112, 3083-3135.

10. Ganem, B. Strategies for innovation in multicomponent reaction design. Accounts Chem. Res. 2009, 42, 463472.

11. Neo, A.G.; Bornadiego, A.; Diaz, J.; Marcaccini, S.; Marcos, C.F. Elusive 2-aminofuran Diels-Alder substrates for a straightforward synthesis of polysubstituted anilines. Org. Biomol. Chem. 2013, 11, 65466555.

12. Bornadiego, A.; Díaz, J.; Marcos, C.F. Synthesis of 4-Aminoxanthones by an Uncatalyzed, Multicomponent Reaction. Adv. Synth. Catal. 2014, 356, 718-722.

13. Bornadiego, A.; Diaz, J.; Marcos, C.F. Regioselective Tandem [4+ 1]-[4+ 2] Synthesis of Amino-Substituted Dihydroxanthones and Xanthones. J. Org. Chem. Marcos. 2015, 80, 6165-6172.

14. Murashige, R.; Hayashi, Y.; Ohmori, S.; Torii, A.; Aizu, Y.; Muto, Y.; Murai, Y.; Oda, Y.; Hashimoto, M. Comparisons of O-acylation and Friedel-Crafts acylation of phenols and acyl chlorides and Fries rearrangement of phenyl esters in trifluoromethanesulfonic acid: Effective synthesis of optically active homotyrosines. Tetrahedron 2011, 67, 641-649.

15. Sagrera, G.; Bertucci, A.; Vazquez, A.; Seoane, G. Synthesis and antifungal activities of natural and synthetic biflavonoids. Bioorg. Med. Chem. 2011, 19, 3060-3073.

16. Al-Zaydi, K.; Borik, R. Microwave assisted condensation reactions of 2-aryl hydrazonopropanals with nucleophilic reagents and dimethyl acetylenedicarboxylate Molecules 2007, 12, 2061-2061.

17. Mkrtchyan, S.; Iaroshenko, V.O.; Dudkin, S.; Gevorgyan, A.; Vilches-Herrera, M.; Ghazaryan, G.; Volochnyuk, D.M.; Ostrovskyi, D.; Ahmed, Z.; Villinger, A.; et al. 3-Methoxalylchromone-A novel versatile reagent for the regioselective purine isostere synthesis. Org. Biomol. Chem. 2010, 8, 5280-5284.

(C) 2019 by the authors. Licensee MDPI, Basel, Switzerland. This article is an open access article distributed under the terms and conditions of the Creative Commons Attribution (CC BY) license (http://creativecommons.org/licenses/by/4.0/). 